\title{
THE IMMOBILIZATION OF WILD ANIMALS AS AN AID TO MANAGEMENT AND CONTROL
}

By Helimut K. Buechner (Department of Zoology, Washington State University, U.S.A.) ; A. M. HARTHOORN (Department of Veterinary Physiology, Makerere College, Uganda); JoHN A. Lock (Department of Pharmacology, Makerere College, Uganda).

It has been realized for a decade that the management of wild animals would be greatly facilitated if a method could be found for their ready capture and handling. The need for such a method has become accentuated in recent years with the increasing interest in wild life, and also with the problems associated with a reduction in the number of wild animals combined with a decrease in the amount of space available for their natural existence. Much work has been done on the catching and marking of animals such as birds, fishes, whales and seals. It is only recently that effective methods are being evolved for the capture of the larger land mammals, such as antelope, for marking and other purposes such as body measurements and weighing, as well as the collecting of samples for pathological or parasitological study. This form of direct control of wild animals is opening up new vistas of wild life management in Africa, and it is thought that the use of these methods may have similar application to wild animals in other regions and parts of the world, both as a means of increasing our knowledge of their habits, ecology and physiology, and in direct management such as capture for transport to other areas, vaccination and occasional therapeutic veterinary interference.

The technique of immobilizing wild animals was introduced into Tanganyika by Michael Grzimek in 1958 to study migration (Gimbel, 1959), and into Uganda by one of us (H. K. B.) to study the territorial behaviour of the Uganda kob (Adenota kob thomasi), using the automatic projectile syringe, and carbondioxide and powder-charged guns evolved by the Palmer Chemical and Equipment Company. These principles have since been extended to some dozen species of animal, the drugs being administered by the Palmer guns, as well as by longbow, cross-bow and several types of modified shotgun and rifle. The medium of injecting the drug has been in most instances the automatic projectile syringe, but also a dart on which the drug dose has been applied in paste, from a syringe equipped with a micrometer screw head, as well as a measured dose of drug introduced into a special projectile fired from a $0 \cdot 22$ rifle. The 
automatic projectile syringe has been used in all instances during the preliminary essay of the exact immobilizing dose per pound body weight, and the type of drug combination suitable for a particular species of animal.

Succinylcholine chloride has been used principally as the muscle relaxant and it has been used on every species immobilized. Parallel essays have been made using neuromuscular blocking agents such as tubercurarine and gallamine triethiodide. Nicotine alkaloids were discarded as unsuitable in the early stages of these experiments.

Succinylcholine was first marketed in the veterinary world as a drug for immobilizing horses and preparing them for surgical procedure and anæsthesia, and we adapted that method for use in wild animals using the zebra (Equus grevyi) for our early experiments. We subsequently adapted dosage rates for the kob, waterbuck (Kobus defassa ugandae), hartebeest (Alcelaphus buselaphus lelwel), and others.

Succinylcholine exerts a muscle paralysing action by causing a persistent depolarization of the motor end plates of the motor nerves to skeletal muscle. Subsequently the drug is broken down rapidly to succinic acid and choline. Its action on a number of horses has been described by Belling and Booth (1955) and also by Stowe (1955) who established the minimum lethal dose for the horse as between $0 \cdot 5$ to $1 \mathrm{mgm}$. per $\mathrm{kgm}$.- - a dose considerably higher than the maximum dose for zebra. The effective dose for bovine-type animals, such as the buffalo, is only about one-eighth of the immobilizing dose reported for the horse.

The disadvantage of succinylcholine is that the dosage rate is for many species, very critical and that consequently in most animals a fairly accurate assessment of the body weight must be made before administration, a factor that introduces grave difficulty in such animals as are rarely seen in the open, like the black rhinoceros, or animals that vary considerably in size such as giraffe. It has many advantages : (a) It is readily soluble in water and the solutions are stable even under East African conditions. (b) It is extremely rapidly absorbed from muscle or peritorial surfaces and there is a relative lack of defined difference in action and dose, when administered by the various routes. (c) Very little is required to relax muscle and therefore small quantities and high dilutions can be used. $(d)$ The lack of unpleasant properties in the solution such as irritation and smell. (e) Its virtually non-poisonous nature when taken by mouth or applied to the skin. $(f)$ Its lack of reaction with agents 
such as atropine when it has to be combined with them for certain species of animals.

In all some five different drugs have been used in these trials on some twelve different species of animal. Besides succinylcholine, a drug mixture where the immobilizing agent is gallamine triethiodide, is showing great promise. Whitetailed deer were successfully immobilized with this drug by Hall and others in 1953. Generally speaking, while succinylcholine is most useful in immobilizing antelope, especially the medium-sized antelope, gallamine triethiodide is finding a special use with those animals that vary greatly in size and weight. The onset of paralysis when using this drug is somewhat slower and the action can be readily reversed with an antidote.

Gallamine triethiodide is a synthetic substance which has muscle relaxing properties similar to that of d-tubocurarine, but is more useful for immobilizing purposes, mainly due to its greater solubility. Muscle relaxation is also obtained more quickly with gallamine. Its action is to some extent reversible by anti-cholinesterase agents so that the results from overdosage may be more easily prevented. It affects the myoneural junction by preventing the access of acetylcholine to the motor end plate, thus blocking the transmission of nervous impulses. There appears to be a considerable margin of safety between the dose needed to prevent locomotion and that which would paralyse the muscles of respiration.

With animals such as buffalo and to a certain extent also with other animals, the addition of atropine in strength of some $5 \mathrm{mgm}$. per $100 \mathrm{lb}$. appears to be essential to control secretions which otherwise would be produced in sufficient quantity to cause asphyxiation. Animals that seldom lie down such as the African elephant seem to require relatively larger doses and more thorough immobilization, before going down than, say, the hippopotamus whose legs become unable to support him while the rest of the body is still quite mobile. The fact that the dose for most of the animals which were immobilized fell into a definite pattern, suggests that the results obtained in Africa can be of direct application to animals in other parts of the world.

The use of this immobilizing technique in its application to wild animals is twofold : firstly, as a means of increasing our knowledge of their habits and physiology ; secondly, as a means of direct management. 
Under the first heading comes the study of certain aspects of animal behaviour such as movement pattern and migration cycles. The movements of wild animals, especially the antelope of the plains, seem deeply ingrained in the species and may have been inculcated very many years ago in connection with seasonal variation in food supply, the search for minerals, and reproduction.

An accurate and detailed knowledge of the movement patterns of these animals is essential for a number of reasons. A knowledge, for instance, of the extent of the seasonal migrations of animals in any one area is a prerequisite for the establishment of the boundaries of animal sanctuaries and National Parks. It is at present a matter for doubt if the migration cycles of animals can be interfered with, without the ultimate destruction of the animals in question. Until the full implication of these wanderings is known for the various species, the boundaries of geographical areas set aside for animal habitat should either include the migration routes or else provide corridors for their accomplishment. The markings of animals by immobilization and tagging is of considerable assistance in the attainment of this end. Ultimately, no doubt, the provision of essential minerals and water may render the present movements of animals unnecessary, and work on these lines is in progress. The territorial behaviour of animals, such as kob, may have important implications in their reproduction, and the marking of these animals has contributed to its study.

Another way in which the immobilization and marking of wild animals may contribute to essential knowledge is by indicating weight gains and incremental rates. It is possible to measure and photograph animals during the time they are immobilized and even to weigh them. These animals may be recaptured on a later occasion and their growth and weight gains established in their natural surroundings. These results, especially if they can be correlated with those of members of the same species kept under experimental conditions, can give valuable indication of these species' value as food converters. The marking of a large proportion of the animals in certain herds and districts will indicate the rates of increment and turnover for that species, and thus also their power of reproduction and regeneration.

The decrease in the number of animals, such as antelope, in Africa, makes it undesirable to eradicate herds of them on contested land. Projects are now on foot to move several of such herds from farm land to alternative accommodation on marginal 
land, where they may continue to exist indefinitely. It is hoped and expected that, with the increase of efficiency of the immobilizing techniques, this method rather than those of destruction may be used to an ever-increasing extent. In the first place it is being used to move animals of species that are in actual danger of extermination. Later it may be extended as an alternative to slaughter, to all those animals that have value to mankind, be it as providers of meat under controlled cropping schemes, as a tourist attraction, or for their ethical value and the part they play in human destiny. Here we would like to quote Sir Julian Huxley, who wrote in the Sunday Times under "The Goal of Fulfilment "- "We must combat everything that threatens the variety of interest needed for human fulfilment-the extermination of wild life, over mechanization, the boredom of mass-production and conformity, the spoiling of nature beauty, the destruction of cultural traditions."

In a yet more positive context these methods may be employed to introduce or reintroduce wild animals where none exist at present. In many parts of Africa, where wild antelope have been exterminated over large tracts of land, there is a growing desire among farmers to have their land restocked with selected wild ungulates. 'This is partly for their ethical value but more for the economic value of these animals, usually as providers of meat. Modern knowledge of the food habits of wild ungulates has demonstrated that many, owing to their browsing habits, do not compete directly with cattle, so that hitherto unused bush and scrub may be utilized. Other animals are appreciated for the quality of their meat and their ability to convert vegetation into meat. There is a growing realization also of the capacity of animals, such as giraffe, to prevent bush encroachment.

The present techniques for immobilizing animals will undoubtedly be improved and fresh methods evolved. Undoubtedly these improvements in management will assist in the opening up of new aspects of the preservation of wild animals.

We are grateful to both the Game Departments and the National Parks of Uganda and of Kenya for their assistance and co-operation. We are indebted to Messrs. Burroughs Wellcome and Co. (East Africa) for donation of the succinylcholine chloride, Messrs. May and Baker, Ltd., for the gallamine triethiodide, Messrs. Roche Products for the prostigmine, and to the Palmer Chemical and Equipment Company, Incorporated, Atlanta, Georgia, for gifts of equipment. This project has been assisted by a grant-in-aid of research made by the Fauna Preservation Society, London. 


\section{LITERATURE CITED}

Belling, T. H., and N. H. BootH, 1955. Studies on the pharmacodynamics of succinylcholine chloride in the horse. Jour. Am. Vet. Med. Association, $126(934), 37-42$.

Buechner, H. K., A. M. Harthoorn, and J. A. Lock, 1960a. Control of African wild animals. Nature, 185 (4705), $47-48$.

1960b. Recent advances in field immobilization of large mammals with drugs. Transactions of the North American Wildlife Conference (in press).

Grmbex, H., 1959. Michael Grzimek. Jour. Wildl. Mgt., 23 (3), 368-369.

HaLL, T. C., E. B. TAFT, W. H. Baken, and J. C. Aub, 1953. A preliminary report on the use of Flaxedil to produce paralysis in the white-tailed deer (Odocoileus virginianus borealis). Jour. Wildl. Mgt., 17 (4), 516-520.

Stowe, C. M., 1955. The curariform effect of succinylcholine in the equine and bovine species-a preliminary report. The Cornell Veterinarian, $45(2)$, 193-197.

[The article above is based on a paper by the same authors presented to the International Union for Conservation of Nature and Natural Resources, Technical Meeting, Poland, June, 1960.-ED.] 